\title{
Spatial Hedonics and the Willingness to Pay for Residential Amenities
}

\author{
Kenneth A. Small and Seiji Steimetz \\ University of California at Irvine (ksmall@uci.edu) \\ and \\ California State University at Long Beach (ssteimet@csulb.edu)
}

February 19, 2007

\begin{abstract}
Housing rents may be influenced by characteristics of nearby properties, an effect captured by spatial autoregression in a hedonic rent equation. We investigate the implications of spatial autoregression for measuring the marginal welfare effects due to a change in a residential amenity such as air quality. We show that if spatial price interdependence arises from technological spillovers, such that utility depends directly on neighboring property values, then the welfare change is given by the reduced form of the autoregressive model, effectively applying a "spatial multiplier" to the relevant implicit price. If instead spatial interdependence arises from merely pecuniary spillovers, as is commonly supposed in motivating spatial autoregression, then no spatial multiplier on implicit prices is called for in computing welfare; but it is then especially important to use the autoregressive model to measure those implicit prices.
\end{abstract}

Keywords: spatial autocorrelation, spatial lag, welfare, willingness to pay, hedonic price function

Acknowledgment: We are grateful to Luc Anselin, Jan Brueckner, Michael Greenstone, Wade Martin, Francisco Martinez, Jeffrey Zabel, and participants at the Stanford Environmental Economics Workshop for comments on earlier drafts. 


\title{
Spatial Hedonics and the Willingness to Pay for Residential Amenities
}

\author{
Kenneth A. Small and Seiji Steimetz
}

It has long been known that residential property values or rental prices depend on prevailing levels of residential amenities or local public goods and that under certain circumstances, that dependence provides a way to measure households' willingness to pay for such amenities or public goods. Pioneering studies focused on air pollution, for example Ridker and Henning (1967) and Freeman (1974). ${ }^{1}$ The basic idea is that in competitive equilibrium, individuals equate their marginal willingness to pay to the marginal price of the amenity that they face in the market, which can be measured through hedonic price techniques (Rosen, 1974).

It is also known that housing values are influenced not only by the characteristics of the house in question but by those of the surrounding neighborhood (Can, 1992). Moreover, some characteristics are not readily observed, but their influences may be captured using spatialeconometric techniques. Among these techniques is the use of "spatial lags", also known as spatial autoregression, in which the prices or characteristics of neighboring properties are explicitly included in hedonic regressions (Anselin, 2002). Using spatially-lagged prices is particularly compelling if neighboring prices are thought to include information on elusive variables like neighborhood quality for which no observable measures can be compiled (Dubin, 1988). For example, the common practice of using "comparables" in real-estate appraisal can be generalized into a spatially-autoregressive hedonic equation (Pace and Gilley, 1998). Case (1991) discusses the distinction between spatial autoregression and other forms of spatial dependence in housing data.

Recent work has shown how spatial econometrics can be applied to a housing-value hedonic equation that includes air pollution variables. Kim, Phipps, and Anselin (2003) hereafter KPA - accomplish this using a spatial lag model, in which the value of a given house is affected by the values of neighboring houses. They note that the full marginal value of an improvement in air quality is then given by a reduced form equation that, in effect, applies a

\footnotetext{
${ }^{1}$ See Freeman (1993), Smith and Huang (1995), and Chay and Greenstone (2005) for summaries of more recent evidence.
} 
"spatial multiplier" to the direct effect of air pollution on property values. The multiplier incorporates the indirect effects that occur because the property in question goes up in value, thereby raising the value of surrounding properties, whose improved values in turn raise the value of the property in question.

It turns out that the combined direct and indirect effect is quite well estimated in the KPA data by ordinary least squares (OLS). This might be viewed as something of a disappointment: although the more sophisticated theory is preferred by statistical tests, it makes little difference to marginal benefits as they measure them. But in fact, the decomposition of the total effect into direct and indirect components is highly informative for policy. The reason is that only under certain rather strong assumptions is this total marginal effect a valid guide to the aggregate benefits of changing the amenity in question (where benefits are defined as aggregate willingness to pay for the change in question ${ }^{2}$ ). Under other assumptions, it is only the direct effect that is relevant.

Thus the advantage of the spatial hedonic methodology for welfare measurement may be less in its theoretically superior estimation properties than in its ability to take into account the mechanism by which the value of a property affects the values of its neighbors. The spatial lag specification enables the analyst to measure the size of price-related externalities and, if they are deemed to be merely pecuniary, to distinguish them from the amenity component of prices and thereby prevent them from confounding the measurement of social welfare.

We demonstrate this claim in the following sections by extending the simple model of Small (1975). That paper, like Freeman (1974), shows that under conventional assumptions, the benefits of a marginal improvement in air quality are correctly measured by summing (over all locations) the marginal price of air quality, $r_{q}$, multiplied by the local change in air quality, $\Delta q$. Here $r_{q}$ is the marginal rental price, $\partial \mathrm{r} / \partial q$, as measured from a hedonic price equation. Note that this sum need not equal the aggregate change in land values, which are affected by generalequilibrium adjustments including relocations, migration, and changes in city size (Freeman, 1971). The result applies only for marginal changes in air quality because nonlinearities in the hedonic equation may cause $r_{q}$ to change as air quality undergoes non-marginal changes.

\footnotetext{
${ }^{2}$ The use of aggregate willingness to pay as a welfare indicator has been debated at length in the literature. The rationale for doing is perhaps best explained by Kaldor (1939), Hicks (1941), and Polinsky (1972).
} 
The intuition behind this earlier result is simple. The marginal price $r_{q}$ measures the annual value to the occupant of a per-unit incremental change in air quality. If the occupant is a renter, that value may be transferred to the property owner through higher rents, but it remains unchanged as a benefit. Similarly, the workings of the property market may transfer these benefits from one property owner to another, but again without changing them.

The extension of this intuition to the hedonic model with spatial lags is more complex. The result now depends on why the value of one property affects that of its neighbors. Suppose first the reason is technological externalities. For example, people may obtain utility from living close to wealthier people or to renters who can afford to live in higher-priced houses; or perhaps higher housing wealth induces better maintenance, which produces a neighborhood-amenity externality. In such cases, the indirect effect identified by KPA represents a true increase in everyone's utility and therefore is properly included in benefits - perhaps offset by higher costs of providing public services. As we discuss later in detail, benefits are then measured by summing terms like $(I-\rho W)^{-1} r_{q} \cdot \Delta q$, where $I$ is the identity matrix and $\rho W$ is a matrix of spatial lags, i.e. a matrix of influences across properties.

Now suppose instead that the reason one property value affects its neighbors is the operation of real estate markets, as suggested for example by Anas and Eum (1984), Pace and Gilley (1998), and Kim, Phipps, and Anselin (2003). Buyers are unable to determine the precise amenities of a given location, so they use the prices of comparable nearby houses as a guide. In that case, the rise in surrounding property values raises the value of the property in question, but it does not produce any increase in the amenity value of living there. So the increased value is just a transfer from the purchaser; surrounding houses produce only a pecuniary externality. Then only the direct effect identified by KPA is part of welfare: it is measured by summing $r_{q}$ $\cdot \Delta q$, just as in the simpler model. In the KPA results, this direct effect is less than half as large as the total effect. Thus under the assumption of only pecuniary externalities, using a model without spatial lags overestimates benefits, whereas the spatial-lag model identifies the correct measure. We suggest that this is an important contribution of the spatial lag model for hedonic propertyvalue estimation

Our strategy is to proceed with the example of a change in air quality and decompose its impact into technological and merely pecuniary effects. We first do this within the traditional analysis (i.e., with no spatial lags), where the decomposition is not very interesting because the 
pecuniary effects cancel out upon aggregation. We then apply the same idea in the spatial-lag analysis.

In order to focus on the issue of interpreting results for a spatially lagged dependent variable, we assume that is the only spatial phenomenon present. Thus we assume that no spatially lagged independent variables enter the specification. We also choose an application in which the additional econometric complication of spatial autocorrelation in the error term is assumed absent, although in practice spatial autocorrelation may be difficult to distinguish from spatial lag. ${ }^{3}$

\section{Traditional Hedonic Analysis}

We first consider the case where there is no spatial interaction among housing prices. Consider a metropolitan area with residential locations indexed by $i$. Let $q_{i}(\theta)$ measure the air quality associated with location $i$, which depends on some index of overall pollution-abatement measures, $\theta$ (taken to be a scalar for expositional simplicity). We assume that $d q_{i} / d \theta>0$. The rental price of a residence at $i$ is $r_{i}=r\left(x_{i}, q_{i}\right)$ where $x_{i}$ is a vector of characteristics of that residence and $r(\cdot)$ is a hedonic rent function. Assuming air quality is considered desirable by everyone, the rent gradient with respect to it, $r_{q i} \equiv \partial r_{i} / \partial q_{i} \equiv \partial r\left(x_{i}, q_{i}\right) / \partial q_{i}$, is everywhere positive. Landlords in the area receive rental revenues $R \equiv \sum_{i} r\left(x_{i}, q_{i}\right) .^{4}$

Residents derive utility from a numeraire $\operatorname{good} z$, housing characteristics, and air quality. Thus when deciding where to live, a resident $i$ with income $y_{i}$ maximizes utility $u_{i}(z, x, q)$ subject to budget constraint $z+r(x, q)=y_{i}$. Consumers need not have identical utility functions. Assuming that a continuum of values of $\mathrm{x}$ and $\mathrm{q}$ are available in the market, we can represent this decision process as:

\footnotetext{
${ }^{3}$ Kelejian and Prucha (1998) provide a general estimation procedure when both spatial autocorrelation and spatial lags are present. Greenbaum (2002) provides an example where the two can be distinguished econometrically.

${ }^{4}$ If houses are owner-occupied, $r_{i}$ is the implicit rental value of the residence, paid by the household (in its role as consumer) to itself (in its role as property owner). See, for example, Mills and Hamilton (1994), pp. 199-206.
} 


$$
\underset{z, x, q, \lambda}{\operatorname{Max}} \Lambda_{i} \equiv u_{i}(z, x, q)+\lambda \cdot\left[y_{i}-z-r(x, q)\right] .
$$

Assuming an interior solution, one of the first-order conditions yields:

$$
\frac{u_{q i}}{\lambda_{i}}=r_{q i}
$$

where $u_{q i} \equiv \partial u_{i} / \partial q$ is the marginal utility of pollution for individual $i$ and $r_{q i} \equiv \partial r_{i} / \partial q$ is the implicit price of pollution at the location chosen by this individual, both derivatives being evaluated at the solution values which we denote $\left(z_{i}, x_{i}, q_{i}, \lambda_{i}\right)$. The properties of Lagrangian multipliers imply that $\lambda_{i}$ is the marginal utility of income. Equation (2) shows that residents will choose a location where the marginal amenity value of pollution just equals its implicit marginal price. Inserting the full solution to (1) into the utility function yields indirect utility $V_{i}$, a function of $y_{i}, \theta$, and all the parameters of the rent function $r(\cdot)$.

How much would residents and landlords be willing to pay for a marginal increase in the abatement parameter $\theta$ ? Using (2) and recalling that $q_{i}$ is a function of $\theta$, the marginal willingness to pay by the resident at location $i$ is:

$$
W_{i \theta} \equiv-\left.\frac{d y_{i}}{d \theta}\right|_{d V_{i}=0}=\frac{d V_{i} / d \theta}{\partial V_{i} / \partial y_{i}}=\frac{u_{q i}}{\lambda_{i}} \frac{d q_{i}}{d \theta}-\frac{d r_{i}}{d \theta} .
$$

where the total derivatives of $V_{i}$ and $r_{i}$ include general-equilibrium effects arising in the land market. Aggregating over all residents and applying (2),

$$
W_{\theta} \equiv \sum_{i} W_{i \theta}=\sum_{i} r_{q i} \frac{d q_{i}}{d \theta}-\sum_{i} \frac{d r_{i}}{d \theta} .
$$

The first term on the right-hand side of equation (4) shows the increased amenity value of the improvement, which we may call a technological effect because it results from the direct effect of pollution on utility. The second term shows the rent increase induced by the improvement, a 
pecuniary effect because it shows up as a benefit to landlords. Aggregate rents increase by the marginal amount:

$$
R_{\theta} \equiv \sum_{i} \frac{d r_{i}}{d \theta} .
$$

Therefore the rent changes, $d r_{i} / d \theta$, cancel in the aggregate benefits to all residents and landlords:

$$
B_{\theta} \equiv W_{\theta}+R_{\theta}=\sum_{i} r_{q i} \frac{d q_{i}}{d \theta} .
$$

Equation (6) shows that the social benefit of the improvement depends only on the technological effects. Part or all of these benefits might be captured by landlords, but the total level of the benefit is unaffected by such rent adjustments. ${ }^{5}$

A special case of (6) is when abatement is uniform across all locations and measured in the same units as air quality. We then have $d q_{i} / d \theta=1$ for every $i$, and

$$
B_{\theta}=\sum_{i} r_{q i} .
$$

\section{Hedonic Analysis with Spatial Lags}

We now allow for the possibility that rent at location $i$ depends on rents at other locations. We do so by writing $r(\cdot)$ in (1) as

$$
r_{i}=r\left(x_{i}, q_{i}, r_{-i}\right)
$$

\footnotetext{
${ }^{5}$ We abstract from any cost savings that the landlord may enjoy, such as less-frequent painting or air-filter replacement. Equation (6) applies only to a marginal air-quality improvement, for which the effects of any induced relocations cancel out because of the envelope theorem applied to each household. It corresponds to the "Stage 1" and "Stage 2" effects described in Bartik (1988, pp. 176-177). Bartik goes on to show that with non-marginal airquality improvements, relocations can add further benefits (a "Stage 3" effect).
} 
where now we are explicit that $x_{i}$ and $q_{i}$ are characteristics of location $i$, and where $r_{-i}$ is a vector of rents at locations other than $i$. At least two reasons for such dependence can be postulated, as already noted, and each leads to a different result. The first is that inter-location rent dependence results from market imperfections, for example when a renter (or buyer) uses rents (or prices) of nearby houses to infer the unknown equilibrium market value of the house being purchased. Thus they are merely pecuniary externalities across locations, affecting utility only through the budget constraint. The second is that residents derive utility from living near high-value properties, for example because people take better care of a house that accounts for a bigger part of their wealth. In that case there is a technological externality across locations, affecting utility directly. (If every desirable trait of a neighborhood could be measured and included in the empirical specification, such technological spillovers could be measured explicitly; but in practice some are likely to be captured instead by spatial-lag effects.) We treat each of these two cases in turn.

\section{Pecuniary Spillovers}

If the dependence of $r_{i}$ on $r_{-i}$ is pecuniary, the Lagrangian problem for household utility maximization is the same as before, and so is condition (2). When we calculate $W_{i \theta}$, the change in rent $d r_{i} / d \theta$ now reflects the entire process of adjustment of rents to pollution abatement, including the indirect effects via pollution's influence on values of nearby locations. Nevertheless, the results of equations (3)-(7) still hold.

Most importantly, the partial derivative $r_{q i}$ entering (7) is that of the function $r(\cdot)$ in (8), holding the values of $r_{-i}$ constant. In other words, in computing aggregate benefits, one continues to use the implicit marginal price of pollution as measured by the fully specified hedonic model with spatial lags. No additional terms related to changes in prices of adjacent locations are necessary because they enter $W_{\theta}$ and $R_{\theta}$ with equal and opposite magnitudes, and thus cancel. Such terms would tend to be positive and so including them would overestimate the benefits of pollution abatement.

Furthermore, if the true rent function is (8) but one omits the spatial lags when estimating it, then the measured gradients $r_{q i}$ will most likely be overestimated in magnitude because of 
positive correlation between $q_{i}$ and the most influential components of $r_{-i}$. Using such measured gradients in (7) would therefore also overestimate aggregate benefits.

Note that the preceding analysis illustrates the importance of the spatial-hedonic approach rather than dismisses it. If spatial effects influence the pollution coefficient, failing to control for them will bias estimates on the direct effect of pollution reduction. This will, in turn, bias the estimates of the welfare gain from a marginal improvement in air quality. To summarize, spatial-hedonic methods improve welfare estimates by "purifying" them of pecuniary effects. Incorporating these effects into welfare estimates will, however, defeat this improvement.

\section{Technological Spillovers}

Now suppose the reason for spatial-lag effects is that residents derive utility from unobservable characteristics that are associated with their neighbors' rent expenditures. For example, people may value the additional maintenance that is undertaken on a neighbor's property when that property's asset value, reflected in its rent, is higher. Or they may perceive that the prestige value of their location depends on property values in the surrounding neighborhood. We thus postulate that utility at $i$ depends on a vector of neighboring rents, $r_{-i}$. This is similar to what Brueckner (2002) calls a "spillover model" in the context of local governments. ${ }^{6}$ The residential location problem is then

$$
\underset{z, x, q, r_{-}, \lambda}{\operatorname{Max}} \Lambda_{i}=u_{i}\left(z, x, q, r_{-i}\right)+\lambda \cdot\left[y_{i}-z-r\left(x, q, r_{-i}\right)\right]
$$

First-order condition (2) still applies. In addition, residents choose the vector of neighboring property values, $r_{-i}$; for example, individuals may prefer a "more prestigious" location among otherwise identical houses. That yields additional first-order conditions

$$
\frac{1}{\lambda_{i}} \frac{\partial u_{i}}{\partial r_{-i}}=\frac{\partial r_{i}}{\partial r_{-i}}
$$

\footnotetext{
${ }^{6}$ See the discussion of Brueckner's terminology in Anselin (2002, p. 249).
} 
where the derivatives with respect to $r_{-i}$ are vectors. This equation shows that the marginal amenity value obtained from each neighboring property's rent is balanced by the increment in own rent required to acquire it through location choice. Note that as in virtually all hedonic models, we assume a sufficient number of choices that the level of each "characteristic", including the value of a nearby property, can be chosen from a continuum while holding constant the characteristics of the house being purchased. The possible failure of this assumption is, of course, a limitation of hedonic analysis.

The marginal benefit to each resident from a change in abatement parameter $\theta$ is now a more complicated version of (3):

$$
W_{i \theta} \equiv \frac{d V_{i} / d \theta}{\lambda_{i}}=\frac{u_{q i}}{\lambda_{i}} \frac{d q_{i}}{d \theta}+\frac{1}{\lambda_{i}} \frac{\partial u_{i}}{\partial r_{-i}} \frac{\partial r_{-i}}{\partial q_{-i}} \frac{d q_{-i}}{d \theta}-\frac{\partial r_{i}}{\partial q_{i}} \frac{d q_{i}}{d \theta}-\frac{\partial r_{i}}{\partial r_{-i}} \frac{\partial r_{-i}}{\partial q_{-i}} \frac{d q_{-i}}{d \theta}
$$

The first two terms on the right-hand side of (11) demonstrate two technological effects: the direct amenity value of improved air quality and the amenity value of technological externalities reflected in an increase in neighboring property values. These benefits are enjoyed at the expense of loss of consumption due to a pecuniary effect: namely, the total increase in rent arising both directly (third term) and indirectly (last term) from the change in policy. The aggregate benefit to residents is then given by the sum over locations of (11), which we can write after applying (2) and (10) as:

$$
W_{\theta}=\sum_{i}\left(r_{q i} \frac{d q_{i}}{d \theta}+\frac{\partial r_{i}}{\partial r_{-i}} \frac{\partial r_{-i}}{\partial q_{-i}} \frac{d q_{-i}}{d \theta}\right)-\sum_{i} \frac{d r_{i}}{d \theta}
$$

where $\partial r_{-i} / \partial q_{-i}$ is a vector of values of $r_{q}$ at neighboring locations, and where $-d r_{i} / d \theta$ denotes the last two terms of (11): that is, $d r_{i} / d \theta$ is the sum of direct and indirect influences of $\theta$ on $r_{i}$. The aggregate benefit to landlords is still given by (5). So when we add the net benefit to residents and landlords, the last terms in (12) and (5) cancel each other, leaving:

$$
B_{\theta}=W_{\theta}+R_{\theta}=\sum_{i}\left(r_{q i} \frac{d q_{i}}{d \theta}+\frac{\partial r_{i}}{\partial r_{-i}} \frac{\partial r_{-i}}{\partial q_{-i}} \frac{d q_{-i}}{d \theta}\right)
$$


In the special case of uniform abatement, where $d q_{i} / d \theta=d q_{-i} / d \theta=1$ for every $i$,

$$
B_{\theta}=W_{\theta}+R_{\theta}=\sum_{i}\left(r_{q i}+\frac{\partial r_{i}}{\partial r_{-i}} \frac{\partial r_{-i}}{\partial q_{-i}}\right)
$$

Equation (14) demonstrates that the welfare effect of a uniform improvement in air quality is now measured by including both the direct and spatially-induced indirect effects of changes in pollution levels on rental prices - presumably both positive when $\theta$ is increased. In the context of a spatial-hedonic specification with spatial lags, the appropriate welfare calculation will apply a "spatial multiplier" to the direct coefficient on the pollution measure. In other words, omitting this spatial multiplier will now yield an underestimate.

\section{Application: Kim, Phipps, and Anselin (2003)}

KPA develop a spatial-hedonic model to estimate the benefits of a uniform reduction in sulfurdioxide $\left(\mathrm{SO}_{2}\right)$ levels in the metropolitan Seoul housing market. Their preferred model is a spatial-lag specification of the following form:

$$
\log r=X \beta_{x}+S \beta_{S}+\rho W \log r+\varepsilon
$$

where $r$ is a vector of housing prices, $S$ is a vector of $\mathrm{SO}_{2}$ levels (which we may view as $-q$ ), $X$ is a matrix of other covariates, $W$ is a spatial-weight matrix, $\varepsilon$ is an error vector, and $\rho, \beta_{S}$, and $\beta_{x}$ are parameters to be estimated. ${ }^{7}$ By convention, the diagonal elements of $W$ are set to zero and the off-diagonal elements are normalized to sum to one by row. This specification is an example

\footnotetext{
${ }^{7}$ Another pollution variable, measuring levels of nitrogen oxides $\left(\mathrm{NO}_{\mathrm{x}}\right)$, shows virtually no explanatory power; we include it among variables in $X$. The components of $\mathrm{NO}_{\mathrm{x}}$ undergo complex chemical reactions in which they are converted to each other and to photochemical oxidants such as ozone, creating a possible explanation for its small explanatory power. More seriously, the values of $\mathrm{SO}_{2}$ and $\mathrm{NO}_{\mathrm{x}}$ are not observed directly for each of the 78 geographical zones used by the authors; instead they are spatially interpolated from 20 observations. As shown by Anselin and Lozano-Gracia (2007), such interpolation may cause the estimated coefficients to be either over- or under-estimated by large magnitudes (roughly a factor of two in their case, as seen in their Tables 7-8).
} 
of the rent function in (8), in which $x_{i}$ is the $i$-th row of matrix $X$ and $q_{i}$ is the negative of the $i$-th entry in vector $S$.

The spatial-lag specification in (15) can be expressed in reduced form, provided $\rho<1$, by solving for $\log (r)$ :

$$
\log r=(I-\rho W)^{-1} Z \beta+v
$$

where $I$ is an identity matrix with dimensions equal to the number of locations, $Z=[X, S]$ is the matrix of all covariates, $\beta=\left(\beta_{x}{ }^{\prime}, \beta_{S}\right)^{\prime}$ is the vector of their coefficients, and $v=(I-\rho W)^{-1} \varepsilon$ is a vector of spatially-weighted error terms. This formulation illustrates a restriction in specifying a spatial lag without error autocorrelation: the explained and unexplained parts of the dependent variable in (16) are required to contain the same spatial transformation, $(I-\rho W)^{-1}$.

From (16), the marginal effects on prices of a change in $S$ is given by the matrix

$$
\frac{d \log r}{d S}=\beta_{S} \cdot(I-\rho W)^{-1}
$$

where both $\log (r)$ and $S$ are vectors. In the case of a uniform reduction in $S$ by amount $d \theta$, the total effect on price at each location is given by the vector

$$
\frac{d \log r}{d \theta}=\beta_{S} \cdot(I-\rho W)^{-1} \cdot(-\mathbf{1})=-\beta_{S} \cdot\left(\frac{1}{1-\rho}\right)
$$

where $\mathbf{1}$ is a vector of ones and the last step uses the fact that the row sum of $(I-\rho W)^{-1}$ is equal to $(1-\rho)^{-1}$ due to the row-normalization of $W$ (see KPA, note 14 , for the proof). KPA call the term in parentheses a "spatial multiplier." Rewriting (18) in terms of the price itself instead of its logarithm,

$$
\frac{d r_{i}}{d \theta}=-\beta_{S} r_{i} \cdot\left(\frac{1}{1-\rho}\right)
$$


This expression corresponds to the term in parentheses in (14), which reflects the direct and indirect price changes induced by the uniform pollution reduction. The direct effect, $-\beta_{S} r_{i}$, estimates the amenity value of a marginal air-quality improvement at each location, while the indirect effect is $-\beta_{S} r_{i}$ multiplied by $\left[(1-\rho)^{-1}-1\right] \equiv \rho /(1-\rho)$.

Table 1 provides a selection of KPA's coefficient estimates from a "spatial two-stage least squares" (S-2SLS) regression, along with estimates from an ordinary least-squares regression (which does not control for spatial effects). ${ }^{8}$ Both regressions are performed in semi$\log$ form. The estimate of the spatial-lag parameter $\rho$ in the S-2SLS column implies a spatial multiplier of $1 /(1-\rho)=2.22$; for OLS, the multiplier is of course 1.0. The last row computes the right-hand side of (18), which differs little between the spatial-lag and OLS regressions.

Table 1

\begin{tabular}{|l|c|c|}
\hline & S-2SLS & OLS \\
\hline $\mathrm{SO}_{2}$ Coefficient $\left(\beta_{S}\right)^{\mathrm{a}}$ & -0.00651 & -0.0151 \\
\hline Spatial-Lag Coefficient $(\rho)$ & 0.549 & 0 \\
\hline$B_{S} /(1-\rho)$ & -0.0144 & -0.0151 \\
\hline
\end{tabular}

${ }^{\mathrm{a}}$ Units: (millions of won)/(parts per billion); \$US1 1200 won.

KPA use (19) to calculate the marginal benefit of a uniform reduction in $\mathrm{SO}_{2}$ levels in Seoul. They obtain an average of US $\$ 2,333$ per household (1.50 percent of average house price) for a 4 percent reduction in ambient SO2 concentrations (an average reduction of 1.27 parts per billion). The analysis in the preceding sections demonstrates, however, that the appropriate welfare measure depends critically on the underlying assumption about whether the spatiallyinduced price effects change the amenity value of each location. If the spatial multiplier captures purely pecuniary externalities, then (19) overstates the benefits of a uniform air-quality improvement by a factor of $1 /(1-\rho)$, or 2.22 in this case; simply using the OLS estimates would

\footnotetext{
${ }^{8}$ These estimates are taken from KPA (2003), Table 2, using the robust form of S-2SLS. Each parameter estimate shown here is statistically significant at a significance level of $10 \%$ or less. The purpose of the S-2SLS procedure is to correct for endogeneity of $\rho W \log (r)$ in (15); the instruments used are $W Z$, one example of a procedure proposed by Kelejian and Prucha (1998).
} 
overstate the benefits by about the same amount. ${ }^{9}$ On the other hand, if the spatial multiplier captures technological externalities, then (19) provides the appropriate welfare measure and omitting the multiplier would understate the benefits of the improvement.

In this particular example, the results ignoring spatial lags (OLS) predict the combined direct and indirect welfare effects quite closely. A similar but less close coincidence of predictions occurs in the study of marginal willingness to pay for ozone and particulate reduction in the Los Angeles region by Anselin and Lozano-Gracia (2007), even when other econometric complications are accounted for. ${ }^{10}$ Of course, such a coincidence depends on the specific data set, and accounting for spatial lags may be important for isolating a number of factors influencing welfare measurements. We argue that one of the most important of these is the ability to control for indirect pecuniary effects when pecuniary but not technological externalities are present.

\section{Conclusion}

Air quality is one residential amenity that can be valued using hedonic price techniques. Freeman (1974) and Small (1975) show that the marginal benefit of a uniform improvement in air quality is correctly measured from traditional hedonic housing-price models by simply summing over the "rent gradient". The more recent development of spatial-hedonic housingprice models, however, introduces a spatial dimension to this gradient. If there is a change that influences all prices simultaneously, such as a uniform pollution reduction, what role does this spatial dimension play in measuring the welfare effects arising from the change?

We demonstrate that the answer depends critically on whether these spatial effects are pecuniary or technological. If reduced pollution increases my neighbors' property values,

\footnotetext{
${ }^{9}$ It is common to find that OLS overstates marginal effects of variables in the presence of spatial lags. For examples in labor economics and public finance, see Greenbaum (2002) and Case, Rosen and Hines (1993).

${ }^{10}$ In Anselin and Lozano-Gracia's model that does not account for possible endogeneity of the pollution variables due to interpolation, $\rho$ is estimated to be 0.3314 (Table 4, column "LAG"). Thus the multiplier on the direct welfare measure is 1.50 , whereas the welfare measure computed by OLS exceed the direct welfare measure by a ratio of 1.30 in the case of ozone and 1.47 in the case of total suspended particulates (calculated form top rows of Tables 7 and 8, columns "OLS" and "LAG Direct"). When they add controls for endogeneity of the interpolated values in the pollution variables, estimated $\rho$ is 0.3266 for a multiplier of 1.49 (Table 4, column "LAG-end"); whereas the ratios of the welfare estimates computed without spatial lags to the direct effects computed with spatial lags is 1.39 for ozone and 1.40 for particulates (their Tables 7 and 8, top rows, columns "IV" and "Lag-end Direct").
} 
thereby increasing the value of my house, but does not further improve the amenity value of my house, then the spatial effect is pecuniary and, therefore, welfare-neutral. If, on the other hand, I derive increased utility from my neighbors' rise in property values, then the spatial effect is technological and is appropriately included in welfare analysis. In the former case, the direct coefficient on pollution produces the correct measure, whereas in the latter case the application of a "spatial multiplier" produces the correct measure.

We have not attempted to provide a means to determine whether spatial autocorrelation is caused by technological or pecuniary externalities. Presumably doing so requires data on the mechanism underlying the spatial price interactions. For example, data on the condition of neighboring houses could identify a pathway from neighboring house prices to spillover amenities; while data on actual transactions could identify a pathway involving price comparisons. Pace et al. (1998) provide an example of the latter approach, in which the authors also take advantage of temporal information on which transactions preceded other ones. In the absence of some such means of measuring the externalities directly, a researcher would have to rely on theoretical reasoning to decide which interpretation of hedonic results is more reasonable.

It is because spatial-hedonic methods are capable of analyzing both cases that we argue their superiority over traditional hedonic methods. The spatial-autoregression model in particular has the advantage that it extracts information about technological spatial spillovers if they are present. Practitioners such as KPA make a valuable contribution in this respect by providing all of the essential information required for welfare analysis in the presence of either pecuniary or technological externalities. 


\section{References}

Anas, Alex, and Sung Jick Eum, Hedonic analysis of a housing market in disequilibrium, Journal of Urban Economics, 15 (1984), pp. 87-106.

Anselin, Luc, Under the hood: issues in the specification and interpretation of spatial regression models, Agricultural Economics 27 (2002) 247-267.

Anselin, Luc, and Nancy Lozano-Gracia, Errors in variables and spatial effects in hedonic house price models of ambient air quality, Working Paper 2007-01, Spatial Policy Analysis Research Consortium, University of Illinois (2007). http://www.sal.uiuc.edu/sparc/research/workingpapers.

Bartik, Timothy J., Measuring the benefits of amenity improvements in hedonic price models, Land Economics 64 (1988) 172-83.

Brueckner, Jan K., Strategic interaction among governments: an overview of empirical studies, International Regional Science Review, 26 (April 2003) 175-188.

Can, Ayse, Specification and estimation of hedonic housing price models, Regional Science and Urban Economics 22 (1992) 453-474.

Case, Anne C., Spatial patterns in household demand, Econometrica, 59(4) (July 1991) 953-965.

Case, Anne C., Harvey S. Rosen, and James R. Hines, Jr., Budget spillovers and fiscal policy interdependence, Journal of Public Economics, 52 (1993), pp. 285-307.

Chay, Kenneth Y., and Michael Greenstone, Does air quality matter? Evidence from the housing market, Journal of Political Economy 113 (2005) 376-424.

Dubin, Robin A., Estimation of regression coefficients in the presence of spatially autocorrelated error terms, Review of Economics and Statistics 70 (1988) 466-474.

Freeman, A. Myrick III, Air pollution and property values: a methodological comment, Review of Economics and Statistics 53 (1972) 415-416.

Freeman, A. Myrick III, On estimating air pollution control benefits from land value studies, Journal of Environmental Economics and Management 1 (1974) 74-83.

Freeman, A. Myrick III, The measurement of environmental and resource values: theory and methods, Resources for the Future, 1993.

Greenbaum, Robert T., A spatial study of teachers' salaries in Pennsylvania school districts, Journal of Labor Research, 23 (2002), pp. 69-86. 
Hicks, John R., The rehabilitation of consumers' surplus, Review of Economic Studies, 8 (Feb. 1941) 108-116.

Kaldor, Nicholas, Welfare propositions of economics and interpersonal comparisons of utility, Economic Journal, 49 (Sept. 1939) 549-552.

Kelejian, Harry H., and Ingmar R. Prucha, A generalized spatial two-stage least squares procedure for estimating a spatial autoregressive model with autoregressive disturbances, Journal of Real Estate Finance and Economics, 17 (1998), pp. 99-121.

Kim, Chong Won, Tim T. Phipps, and Luc Anselin, Measuring the benefits of air quality improvement: a spatial hedonic approach, Journal of Environmental Economics and Management 45 (2003) 24-39.

Mills, Edwin S., and Bruce W. Hamilton, Urban Economics, HarperCollins, 1994.

Pace, R. Kelley, Ronald Barry, John M. Clapp, and Mauricio Rodriguez, Spatiotemporal autoregressive models of neighborhood effects, Journal of Real Estate Finance and Economics, 17 (1998), pp. 15-33.

Pace, R. Kelley, and Otis W. Gilley, Generalizing the OLS and Grid estimators, Real Estate Economics 26 (1998) 331-347.

Polinsky, A. Mitchell, Probabilistic compensation criteria, Quarterly Journal of Economics, 86 (Aug. 1972) 407-425.

Ridker, Ronald G., and John A. Henning, The determinants of residential property values with special reference to air pollution, Review of Economics and Statistics 49 (1967) 246-257.

Rosen, Sherwin, Hedonic prices and implicit markets: product differentiation in pure competition, Journal of Political Economy 82 (1974) 34-55.

Small, Kenneth A., Air pollution and property values: further comment, Review of Economics and Statistics 57 (1975) 105-107.

Smith, V. Kerry, and Ju-Chin Huang, Can markets value air quality? A meta-analysis of hedonic property value models, Journal of Political Economy 103 (1995) 209-227. 